\title{
Ulk4 regulates GABAergic signaling and anxiety-related behavior
}

\author{
Min Liu', Marie Fitzgibbon², Yanqin Wang (1) ${ }^{1,3}$, Jamie Reilly', Xiaohong Qian ${ }^{4}$, Timothy O'Brien ${ }^{1}$, Steve Clapcote $\mathbb{E}^{5}$, \\ Sanbing Shen ${ }^{1}$ and Michelle Roche ${ }^{2}$
}

\begin{abstract}
Excitation/inhibition imbalance has been proposed as a fundamental mechanism in the pathogenesis of neuropsychiatric and neurodevelopmental disorders, in which copy number variations of the Unc-51 like kinase 4 (ULK4) gene encoding a putative Serine/Threonine kinase have been reported in approximately 1/1000 of patients suffering pleiotropic clinical conditions of schizophrenia, depression, autistic spectrum disorder (ASD), developmental delay, language delay, intellectual disability, or behavioral disorder. The current study characterized behavior of

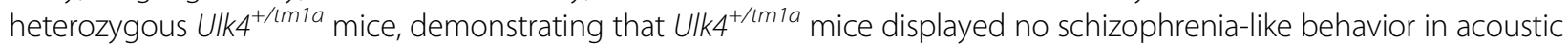
startle reactivity and prepulse inhibition tests or depressive-like behavior in the Porsolt swim or tail suspension tests. However, Ulk4t/tmla mice exhibited an anxiety-like behavioral phenotype in several tests. Previously identified hypoanxious (Atp1a2, Ptn, and Mdk) and hyper-anxious (Gria1, Syngap1, and Npy2r) genes were found to be dysregulated accordingly in Ulk4 mutants. Ulk4 was found to be expressed in GABAergic neurons and the Gad67 $7^{+}$interneurons were significantly reduced in the hippocampus and basolateral amygdala of Ulk4 $4^{+/ t m 1 a}$ mice. Transcriptome analyses revealed a marked reduction of GABAergic neuronal subtypes, including Pvalb, Sst, Cck, Npy, and Nos3, as well as significant upregulation of GABA receptors, including Gabra1, Gabra3, Gabra4, Gabra5, and Gabrb3. This is the first evidence that Ulk4 plays a major role in regulating GABAergic signaling and anxiety-like behavior, which may have implications for the development of novel anxiolytic treatments.
\end{abstract}

\section{Introduction}

Anxiety disorders are a category of mental disorders, including generalized anxiety disorder, panic disorder and phobias, characterized by feelings of anxiety and fear. They are the most common mental illness with an estimated prevalence of approximately $20 \%$ of world population, affecting 69 million of people in the $\mathrm{EU}^{1}$. Recently a meta-analysis was carried out and showed that deletions of 33 hypo-anxious genes were accompanied with increased anxiety, whereas deletions of other 34 hyper-

\footnotetext{
Correspondence: Sanbing Shen (sanbing.shen@nuigalway.ie) or Michelle Roche (michelle.roche@nuigalway.ie)

${ }^{1}$ Regenerative Medicine Institute, School of Medicine, National University of Ireland Galway, Galway, Ireland

${ }^{2}$ Physiology, School of Medicine, Galway Neuroscience Centre and Centre for Pain Research, National University of Ireland Galway, Galway, Ireland Full list of author information is available at the end of the article Min Liu and Marie Fitzgibbon contributed equally to this work.
}

anxious genes were shown to result in decreased anxiety, with both presynaptic and postsynaptic genes involved ${ }^{2}$.

Clinical evidence suggests that altered GABA transmission contributes to the pathophysiology of anxiety disorders in humans. The inhibitory neurotransmitter GABA is synthesized from glutamate by two distinct enzymes, Gad67 (encoded by Gad1) and Gad65 (encoded by $G a d 2)^{3,4}$. The levels of circulating GABA in the central nervous system is largely determined by the $\mathrm{Gad} 67^{+}$cells, as deletion of the Gad1 gene results in $>90 \%$ reduction in basal GABA levels, whereas $\mathrm{Gad} 2^{-/-}$mice expressed normal levels of GABA ${ }^{5,6}$.

Previous studies from our group have shown that Unc51 like kinase 4 (ULK4), a gene encoding a putative Ser/ Thr kinase, is co-expressed in $\mathrm{Gad} 67^{+}$neurons in the cortex and hippocampus of mouse and human brains ${ }^{7}$. In a follow-up study, we showed that ULK4 was deleted in 
$1.2 / 1000$ of patients with developmental delay, language delay, severe intellectual disability, and behavior disorder ${ }^{8}$. Recent studies from our group and others have demonstrated that Ulk4 is involved in cortical development ${ }^{8-10}$, ciliogenesis, and CSF flow ${ }^{11}$ in the brain.

In this study, we performed a series of behavioral tests in the $U l k 4^{+/ t m l a}$ mice, as patients carry one copy of ULK4 gene deletion, and the majority of the Ulk4tmla/tm1a mutant mice die within the first 3 postnatal weeks ${ }^{11}$. We demonstrated that the $U l k 4^{+/ t m l a}$ mice exhibited anxietyrelated phenotype, with no significant alteration in schizophrenia-related behavior such as prepulse inhibition (PPI), acoustic startle reactivity (ASR), or depressionlike behavior of Porsolt swim test (PST) and tail suspension test (TST). This anxiety-related phenotype was accompanied by an imbalanced expression of hypoanxious and hyper-anxious genes, reduced expression of markers for GABAergic interneuron subtypes, decreased Gad $67^{+}$cells in the hippocampus and basolateral amygdala and increased GABA receptors.

\section{Materials and methods \\ Ulk4 hypomorph mice}

The Ulk $4^{+/ t m l a}$ mice were created from ES cell clone EPD0182_4_E12 on C57BL/6N background ${ }^{12}$. The gene targeting was done by inserting a FRT-En2SA-IRES-LacZ$P A$-hBactP-Neo-PA-FRT-loxP cassette into the intron 6 of the $U l k 4$ gene. The construct was designed to truncate the Ulk4 transcription after exon 6 by fusing with En2SAIRES-LacZ-PA containing an En2 splicing acceptance (En2SA) site. We previously shown that low levels of Ulk4 mRNA expression were detected in homozygous Ulk $4^{\text {tmla/tmla }}$ mutants using primers downstream of the exon 6, and this could be resulted from the leaking of the construct and/or alternative splicing, despite the Ulk4 isoforms are largely unknown. The Ulk $4^{\text {tmla/tmla }}$ strain is therefore regarded as a hypomorph model, rather than a null mutant ${ }^{11}$. The genotyping was carried out by polymerase chain reaction (PCR). The wild-type (WT) allele was detected by a $271 \mathrm{bp}$ PCR product using primers Ulk4EndE7For $\quad\left(5^{\prime}\right.$-TAACTTGCTGGACGGATTGCT G-3') and Ulk4EndIn7Rev (5'-TGATCTGTAATCGCAGTGCAGG- $3^{\prime}$ ). The mutant allele gave rise to a $621 \mathrm{bp}$ DNA band using primers Ulk4KOMPKOFor (5'-GAGATGGCGCAACGCAATTAATG-3') and Ulk4KOMPKORev (5'-CTGAGGAGACAATGTAACCAGC$\left.3^{\prime}\right)^{8,11}$.

All experimental procedures were approved by the Irish Department of Health and Children in accordance with Cruelty to Animals Act of 1876 and by the Institutional Animal Care and Research Ethics Committee. The $U l k 4^{+/ t m l a}$ and WT littermates were generated from $U l k 4^{+/+} \times U l k 4^{+/ t m l a}$ mating for behavioral tests and immunohistochemistry. Mice were group-housed according to gender after weaning, and kept at $21 \pm 2{ }^{\circ} \mathrm{C}$ under 12:12 h light dark (lights on from 07.00 to $19.00 \mathrm{~h}$ ). All behavioral experiments were carried out between 10.00 and $17.00 \mathrm{~h}$ from 8 week old. For RNA sequencing, the Ulk4 $4^{\text {tmla/tmla }}$ mice and WT littermates were produced by $U l k 4^{+/ t m 1 a} \times U l k 4^{+/ t m 1 a}$ mating.

\section{Three-chamber sociability and social novelty preference test}

The sociability and social novelty preference test were carried out in a three-chamber apparatus ${ }^{13-16}$. Each animal was placed into the center of the arena and allowed access to all chambers for $10 \mathrm{~min}$. Distance moved and time spent in each compartment was assessed to evaluate general locomotor activity and preference for any compartment. Following this acclimatization period, two identical small cages were placed in the two-side chambers, one containing an unfamiliar stimulus mouse of the same sex, which was randomly assigned to either the right or left chamber of the arena (sociability testing). The test animal was then allowed to freely explore the entire arena for a further $10 \mathrm{~min}$ and behavior recorded. Following this, the test animal was confined to the central chamber, while a second unfamiliar mouse of the same sex to the test mouse was placed into the empty wire cage (social novelty preference). The test animal was then allowed to freely explore the arena for further $10 \mathrm{~min}$ and behavior recorded. Distance moved in the arena, time spent in each chamber, as well as duration and frequency of engaging in investigatory behavior were assessed. All behaviors were evaluated with the aid of EthoVision XT software (Noldus Netherlands).

\section{Novel open field test}

Mice were placed in a novel open field arena $(30 \times 30 \times$ $30 \mathrm{~cm}$ ) for a 20 -min period and behavior was recorded onto a DVD. Distance moved and time spent on grooming were assessed using Ethovision XT software (Noldus Netherlands).

\section{Marble burying test (MBT)}

The MBT is a well-validated screen for anxiety/neophobia-related behavior. This test was carried out as described $^{17,18}$ with minor modifications. A novel test cage contained 15-cm deep wood shavings bedding, with 15 black glass marbles arranged in an equidistant $5 \times 3$ grid on top of the bedding. Animals were placed in the testing arena for $20 \mathrm{~min}$, and the number of buried marbles was recorded and statistically analyzed.

\section{Elevated plus maze (EPM)}

The EPM arena consisted of a wooden apparatus, elevated to a height of $55 \mathrm{~cm}$ above the floor, with two open $(50 \times 10 \mathrm{~cm}$, lux 65$)$ and two closed arms $(50 \times 10 \times 30$ $\mathrm{cm}$, lux 30) extending from a central platform $(10 \times 10$ 
$\mathrm{cm})^{19}$. Mice were placed individually on the central platform facing an open arm and behavior in the arena recorded over a 5-min period. Anxiety-related behavior was analyzed by using Ethovision XT software and the time spent in open and closed arms, and the number of entries by the subjects into the open arms assessed. Locomotor activity was assessed as distance moved over the trial duration.

\section{Histology and immunohistochemistry}

The histology and immunohistochemistry were carried out as previously described ${ }^{8,11}$ in five WT females and five $l k 4^{+/ t m l a}$ females. The primary antibody was mouse antiGad67 (MAB5406, Millipore). The secondary antibody was biotinylated goat anti-mouse (71-00-18, KPL). Gad67 ${ }^{+}$neurons were stained with diaminobenzidine, and imaged under a bright filed microscope (IX41, Olympus) equipped with a camera.

Brain structures were delineated according to the mouse brain atlas ${ }^{20}$. Images were taken at $4 \times$ objective lens, under an Axiovert 40CFL microscope (Zeiss). Cell counting for $\mathrm{GAD}^{+} 7^{+}$neurons was performed on the basolateral amygdaloid complex surrounded by the amygdala capsule (Bregma-1.34-1.70 mm). The mean cell numbers in the outlined basolateral amygdala and hippocampus from 6-10 coronal serial sections/mouse were used for statistical analysis. The X-gal staining was carried out to reveal the Ulk4 expression pattern as the $U l k 4^{+/}$ tm1a strain was an insertional mutation. The staining solution contained $1 \mathrm{mg} / \mathrm{ml} \mathrm{X}$-gal, $5 \mathrm{~mm} \mathrm{~K} 4 \mathrm{Fe}(\mathrm{CN}) 6,5$ $\mathrm{mm} \mathrm{K3Fe}(\mathrm{CN}) 6,2 \mathrm{~mm} \mathrm{MgCl} 2,0.02 \%$ Nonidet P-40, $0.01 \%$ sodium deoxycholate in PBS $^{11}$.

\section{RNA transcriptome analyses and target validation}

RNA was extracted from P12 cortex of one male and two female WT mice and three female Ulk4 $4^{\text {tm1a/tm1a }}$ mutants. Whole-genome RNA sequencing and analyses were performed by BGI and transcriptome was analyzed on the top 1945 differentiated expressed genes as previously described ${ }^{11}$. Quantitative RT-PCR was carried out to validate gene expression of selected Ulk4 targets. Single-strand cDNA was reversely transcribed from cortical RNA of four WT and four $U l k 4^{\text {tm1a/tm1a }}$ mice. Triple qRT-PCR reactions were performed for each primer pair, included PvalbFor 5' ${ }^{\prime}$-TGTCGATGACAGACGTGCTC-3' and PvalbRev 5'-ACCTTCTTCACCTCATCCGG-3' (138 bp); MdkFor 5' $5^{\prime}$-TGAAGAAGGCGCGGTACAATG-3' and MdkRev 5'-ATATCTTGGGCCTGTGGGAGA-3' (186 bp); Gng2For 5'-GATAAGCTGTCGCCCCATGT-3' and Gng2Rev 5'-GGGTTCATTCCTGCTTGGAG-3' (158 bp); Slc38a1For 5'-GCCATGTCTTGGTGACCAT C-3' and Slc38a1Rev 5'-ATCTCCGTCCTGGTTCGT GA-3' (167 bp); Slc38a2For 5'-TTACGGACACGTGGA ATCGG-3' and Slc38a2Rev 5'-
AGTGACGGAACTCCGGATAG-3' (148 bp); Slc38a3For 5'-TGTTCTGTTCCCG

GTACGAC-3' and Llc38a3Rev 5'-GTTGGGGGCGAAGATAACCA -3' (133 bp). Each plate was loaded with house-keeping reaction mixtures and corrected for GapdhFor 5'-CTCATGACCACAGTCCATGC-3' and GapdhRev 5'-CACATTGGGGGTAGGAACAC-3' as cDNA loading. Relative RNA abundance in the $U l k 4^{\text {tmlal }}$ tm1a cortex was calculated using $2^{\Delta \Delta \mathrm{Ct}}$, with average expression level of the corresponding gene in the WT littermates as $100 \%$. The data were presented as mean \pm standard error of the mean (SEM), $n=4$ each, " $p<0.05$.

\section{Statistical analysis}

All data were expressed as mean \pm SEM. Behavioral data were analyzed with two-way ANOVA when both sex and genotype were considered or one-way ANOVA when only one factor was considered. The immunohistochemistry data were analyzed using one-way ANOVA. Data were considered significant when $p<0.05$. For RNA sequencing, expression data were normalized to FPKM (fragments per kilobase of transcript per million mapped reads). The differentially expressed genes were shortlisted with $p$-values of multiple testing and false discovery rates (FDRs), and transcriptome pathways were carried out as previously analyzed ${ }^{11}$.

\section{Results}

Ulk4 $4^{+/ t m 1 a}$ male mice exhibit impaired social novelty preference

Ulk $4^{+/ t m l a}$ mice were comprehensively examined for behavioral changes, as single-copy deletion of the ULK4 gene was identified in patients of neuropsychiatric and neurodevelopmental disorders ${ }^{7,8}$. We tested schizophrenia-related positive or negative symptoms by ASR, PPI, and depression-related behavior using the PST and TST. Ulk $4^{+/ t m 1 a}$ mice exhibited no significant difference from the WT littermates in any of these tests (Supplementary Figs. S1, S2, S3).

We next investigated the sociability and preference for social novelty in three-chamber apparatus (Fig. 1a), as impaired social behavior is common in neurodevelopmental and neuropsychiatric disorders. During the 10min habitation period, $U l k 4^{+/ t m l a}$ mice displayed no zone preference (Fig. 1b). In the subsequent 10-min sociability test, all animals spent significantly $(p<0.01)$ more time in the chamber with a novel con-specific mouse (of the same sex as the test mouse) than in the center zone or the chamber with an empty cage, with no statistical difference between WT and Ulk4 ${ }^{+/ t m l a}$ mice (Fig. 1c). In the social novelty preference test (Fig. 1d), all mice spent significantly increased time in the chamber with a novel mouse than the chamber containing the familiar mouse 

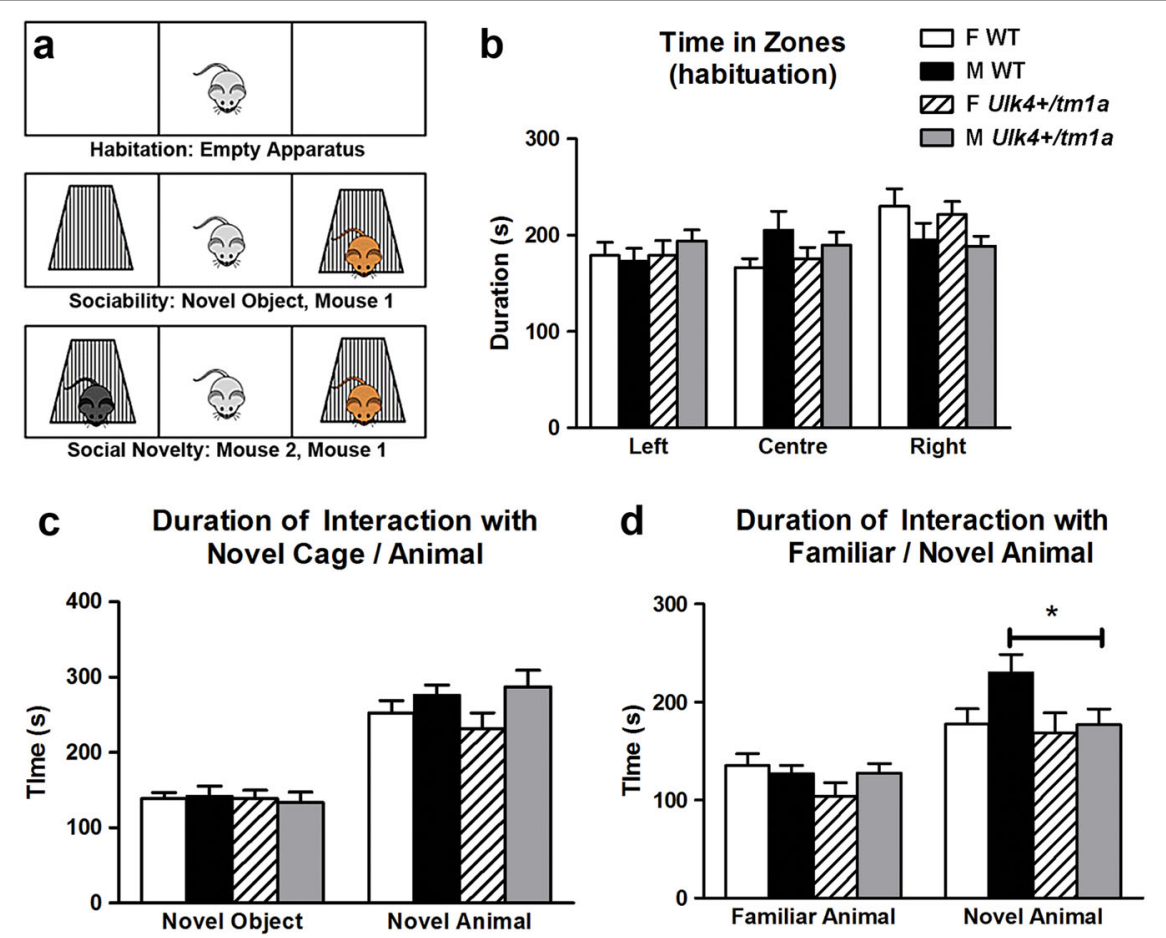

Fig. $1 \mathrm{Ulk4}^{+/ t m 1 a}$ male mice display reduced social novelty preference. a WT male $(n=18)$, WT female $(n=20), U / k 4^{+/ t m l a}$ male $(n=20)$, and Ulk4 ${ }^{+/ t m l a}$ female $(n=14)$ were tested in three-chamber apparatus. b No zone preference during the habitation period $(p>0.05)$. $\mathbf{c}$ No genotype difference in sociability test. All animal spent more time in the arena with the novel stimulus animal vs. time in center or in arena of novel object $(p<$

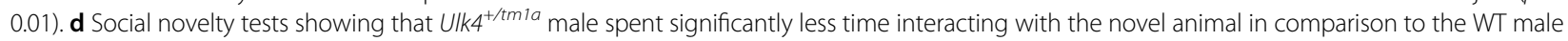
$\left({ }^{*} p<0.05\right)$

$(p<0.01)$. However, $U l k 4^{+/ t m 1 a}$ male spent significantly less time interacting with the novel male mouse in comparison to WT male (Fig. 1d, $p<0.05$ ), an effect not observed in female counterparts. This suggests that Ulk4 mutation may influence social novelty preference of male mice.

Ulk4 $4^{+/ t m 1 a}$ female mice exhibit anxiety-related behavior in the MBT

Repetitive or stereotypical behavior is common in anxiety and ASD. As such we examined duration of grooming behavior as a measure of repetitive activity in open field test. There was no genotype-specific difference on the distance moved during the test $(p=0.23)$, although males (both WT and $U l k 4^{+/ t m 1 a}$ ) displayed lower locomotor activity than corresponding females (Fig. 2a). For grooming behavior, $U l k 4^{+/ t m 1 a}$ male mice displayed more repetition-like activity, with grooming time doubled (77.3 $\pm 6.3 \mathrm{~s}, n=17$ ) when compared to $U l k 4^{+/ t m 1 a}$ female $(39.8 \pm 5.1 \mathrm{~s}, n=13, p<0.01$, Fig. $2 \mathrm{~b})$, whereas WT male mice exhibited no difference in duration of grooming from WT female $(p=0.20)$. Temporal analysis of grooming behavior over the $20 \mathrm{~min}$ trail demonstrated that $U l k 4^{+/ t m 1 a}$ female failed to increase grooming activity in the last $10 \mathrm{~min}$ of the trial when compared to other groups (WT female: $31.63 \pm 4.10 \mathrm{~s}$ vs. $U l k 4^{+/ t m 1 a}$ female: $19.86 \pm 3.18 \mathrm{~s} ; p<0.05)$, and also showed lower rearing activity than WT female (Fig. 2c), suggesting that $U l k 4^{+\prime}$ tm1a females fail to acclimatize the novel arena possibly due to enhanced anxiety-related behavior.

To further evaluate anxiety-like behavior, we carried out the $\mathrm{MBT}^{14,15}$. Ulk $4^{+/ t m 1 a}$ females buried significantly more marbles $(9.6 \pm 1.0, n=13)$ than WT females $(5.9 \pm$ $1.0, n=20, p=0.01$, Fig. $2 \mathrm{~d})$. The $U l k 4^{+/ t m 1 a}$ group as a whole also buried significantly more marbles $(7.8 \pm 0.8$, $n=32)$ than the WT group $(5.6 \pm 0.7, n=38, p=0.03)$. This further confirmed anxiety-like or neophobia-like behavior in $U l k 4^{+/ t m l a}$ mice.

\section{$U l k 4^{+/ t m 1 a}$ mice exhibit anxiety-related behavior on the EPM}

We next carried out EPM test ${ }^{16}$ to further assess anxiety-like behavior in the mutant mice. Female mice exhibited greater locomotor activity on the EPM than males in consistency with the open field test, although this was not genotype-specific (Fig. 3a). Both $U l k 4^{+/ t m l a}$ male 

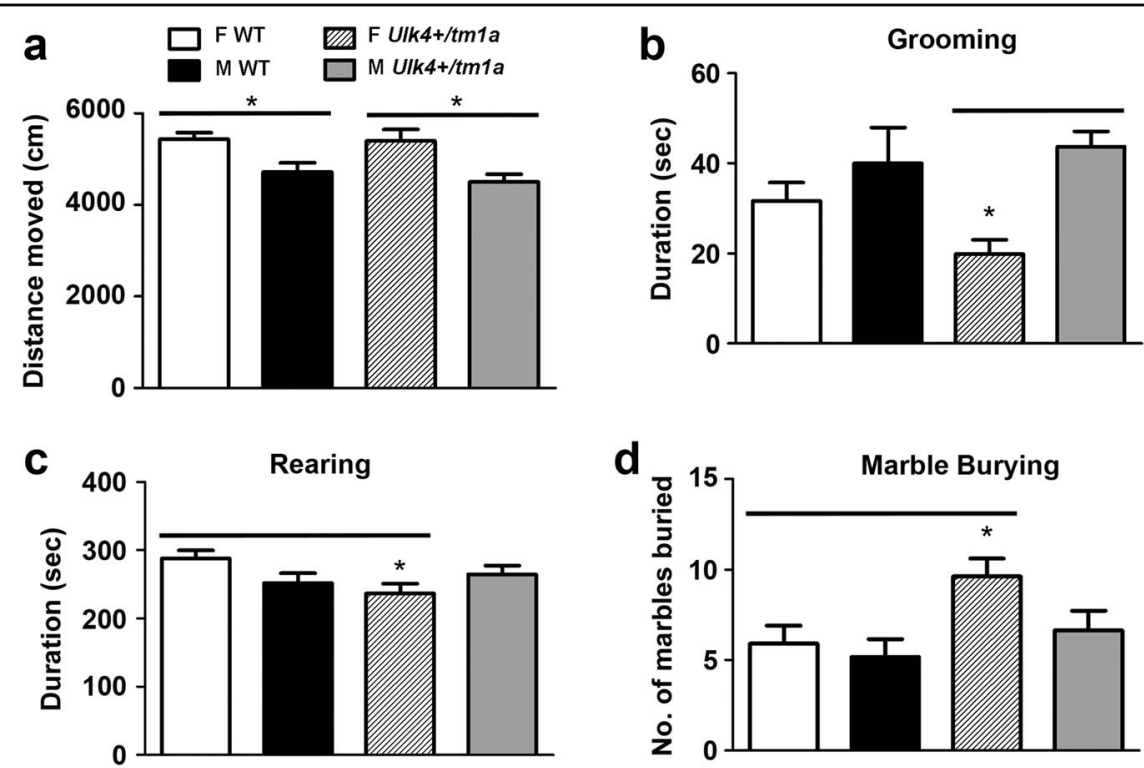

Fig. $2 \mathrm{Ulk4}^{+/ \mathrm{tm} \mathbf{l a}}$ female mice displayed decreased grooming activity, reduced rearing, but increased marble burying. a-c $20 \mathrm{WT}$ female, 18 WT male, $13 \mathrm{Ulk4^{+/tm1a }}$ female, and $17 \mathrm{Ulk4^{+/tmla }}$ male at 2-3-month-old were placed in an open field arena for 20 min. a There was no genotypespecific but gender-associated difference in distance traveled. $\mathbf{b}$ The duration spent on grooming was reduced in U/k4 ${ }^{+/ t m 1 a}$ female $(39.8+5.1, n=13)$

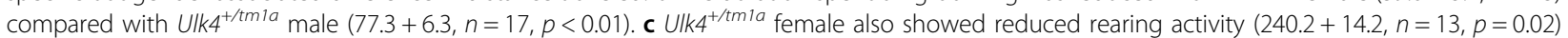
than WT Female $(287.7+12.1, n=20)$. d U $/ k 4^{+/ t m l a}$ female and total Ulk $4^{+/ t m l a}$ mice showed significant increase in marble burying compared to WT female and total WT mice
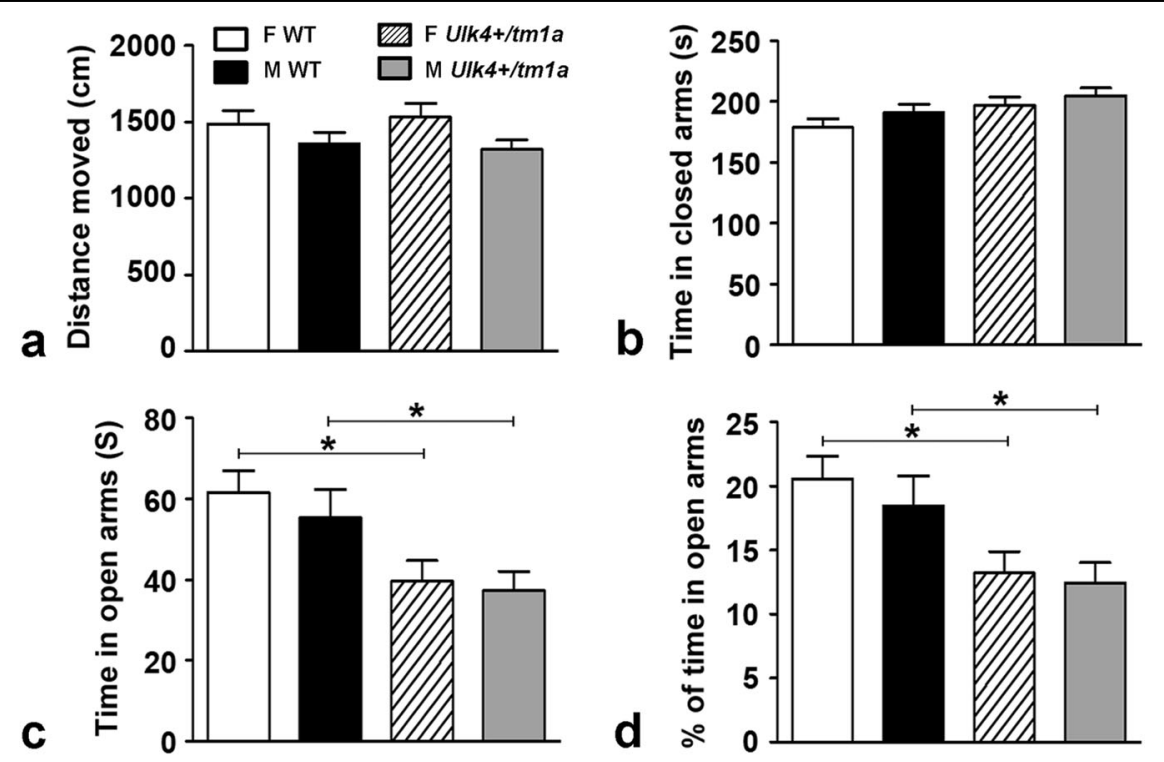

Fig. 3 Both Ulk4 $4^{+/ t m 1 a}$ male and female mice exhibit an increased anxiety on EPM test. a Distance moved; $\mathbf{b}$ time spent in the closed arms; $\mathbf{c}$ time spent in open arms; and $\mathbf{d}$ percentage of time spent in the open arms. Male and female Ulk4 $4^{+/ t m 1 a}$ mice and their WT littermates were subjected to the EPM. Data are expressed as mean \pm SEM; $n=12-19$ per group. ${ }^{*} p<0.05$

and female mice spent significantly less time in the open arms of the EPM than WT counterparts (Fig. 3c, d). Thus, $U l k 4^{+/ t m 1 a}$ mice display an elevated anxiety-like behavior in the EPM.
Ulk4 $4^{+/ t m 1 a}$ mice show fewer Gad67 ${ }^{+}$cells in basolateral amygdala and hippocampus

Neuroanatomic analysis demonstrated normal brain morphology in $U l k 4^{+/ t m 1 a}$ mice, with no schizophrenia- 
related or hydrocephalus-related phenotype (Supplementary Fig. 4). Thus, a single copy of Ulk4 gene deletion does not affect gross neuroanatomy in mice. We previously showed that Ulk4 is expressed in GABA neurons ${ }^{7}$, and altered GABA signaling is implicated in neuropathology of the neuropsychiatric illness. The amygdala and hippocampus are key brain regions associated with anxiety, fear, and learning. We took advantage of lac $Z$ reporter gene in the Ulk4 mutant, performed X-gal staining, and demonstrated the strongest $\beta$-gal activity in the medial part of the basolateral amygdala (Fig. 4a) and in the CA1 region of the hippocampus (Fig. 4h). We subsequently carried out anti-Gad67 staining in 2-month WT and $U l k 4^{+/ t m 1 a}$ mice, and quantified $\mathrm{Gad} 67^{+}$cells (Fig. 4b, c) from comparable sections in the basolateral amygdaloid complex and hippocampus. The data revealed there were $72.9 \pm 6.3 \mathrm{Gad} 7^{+}$cells in the WT basolateral amygdala, with a cell density of $109.8 \pm 13.3$ cells $/ \mathrm{mm}^{2}$ (Fig. 4b, c). In Ulk $4^{+/ t m 1 a}$ mice, $50.9 \pm 1.7{\mathrm{Gad} 67^{+}}^{+}$cells were found in the equivalent region with a cell density of $58.7 \pm 11.1$ (Fig. 4b-e). These data demonstrated a significant reduction of $\mathrm{Gad}^{+} 7^{+}$cells $(p=0.01)$ and cell density $(p=0.02)$ in the amygdala of the $U l k 4^{+/ t m 1 a}$ mice.

In the hippocampus, an average of $146.0 \pm 4.3{\mathrm{Gad} 67^{+}}^{+}$ cells was found on the WT hippocampus, whereas $U l k 4^{+/}$ ${ }^{t m 1 a}$ mice exhibited an average cell number of $110.6 \pm 8.2$ cells (Fig. 4f, g, i, j, $n=5$ each, $p<0.01$ ). This was resulted from general decrease of Gad67 ${ }^{+}$cells in the CA1 (81.2 \pm 3.0 vs. $62.9 \pm 6.1, p<0.05), \mathrm{CA} 2(13.7 \pm 1.9$ vs. $10.3 \pm 0.7$, $p>0.05)$, CA3 $(27.0 \pm 2.8$ vs. $19.0 \pm 0.9, p<0.05)$, and DG $(24.1 \pm 1.2$ vs. $18.4 \pm 1.4, p<0.05)$ of $U l k 4^{+/ t m 1 a}$ vs. WT mice. Therefore, these data demonstrated that $U l k 4^{+/ t m l a}$ mice exhibit reduced numbers of GABAergic neurons in the basolateral amygdala and hippocampus, thus possibly provide the cellular mechanism for the anxiety-like behavior in $U l k 4^{+/ t m 1 a}$ mice.

\section{Reduced expression of markers for GABAergic interneuron subtypes in the Ulk4 ${ }^{\text {tm } 1 a / t m 1 a}$}

GABAergic interneurons are the major inhibitory neurons, with $>12$ distinct subtypes of GABAergic interneurons expressing calretinin (Calb2), somatostatin (Sst), cholecystokinin (Cck), neuropeptide Y (Npy), vasointestinal polypeptide (Vip), choline acetyltransferase (Chat), and $\mathrm{NOS}^{21}$. To systematically evaluate the effect of $U l k 4$ lesion of the various GABA interneuron subtypes, we carried out transcriptome analyses in the WT and Ulk $4^{\text {tmla/tmla }}$ mice by whole-genome RNA sequencing. The expression of Pvalb, Sst, Cck, Npy, and Nos3 were all reduced in Ulk4 mutants (Table 1). Pvalb-expressing cells constitute $39 \%$ of GABA interneurons and are vital for neuronal synchronization. Notably Pvalb was $37 \%$ reduced in Ulk4 mutants $(p=4.33 \mathrm{E}-07, \mathrm{FDR}=$ 9.01E-06). Together these data support a substantial reduction of pre-synaptic GABA interneurons in $U l k 4$ mutants.

\section{Disrupted GABAergic synapse in Ulk4 $4^{\mathrm{tm} 1 \mathrm{a} / \mathrm{tm} 1 a}$ mice}

Analyses of the top 1945 differentially regulated genes (FDR $<0.025, p<0.0033)$ identified "Synapse" pathway $(\mathrm{FDR}=1.51 \mathrm{E}-18)$ with 124 genes significantly altered in Ulk4 mutants. More "Postsynapse" genes $(n=72, \mathrm{FDR}=$ $1.55 \mathrm{E}-12)$ were affected than "Presynapse" genes $(n=26$, $\mathrm{FDR}=1.03 \mathrm{E}-03$ ). KEGG pathway analyses showed that 16 genes in the GABAergic synapse $(F D R=0.016)$ were significantly dysregulated in Ulk4 mutants (Table 1). Whereas Gng7, Gng4, Slc38a3, and Slc38a5 were downregulated in mutants, postsynaptic targets Gabra1, Gabra3, Gabra4, Gabra5, and Gabrb3 genes were significantly upregulated, together with overexpression of Nsf, Slc38a1, Slc38a2, Hap1, Gng2, Adcy9, and Cacna1c. This highlights that $U l k 4^{\text {tmla/tmla }}$ mutation significantly alters both pre-synaptic and post-synaptic GABAergic signaling.

\section{Ulk4 regulates hyper-anxious and hypo-anxious gene expression}

The previous meta-analysis identified 33 hypo-anxious and 34 hyper-anxious genes ${ }^{2}$. The quantitative RNA sequencing showed that three hypo-anxious genes Atpla2 $(\mathrm{FDR}=1.40 \mathrm{E}-114), \quad$ Ptn $\quad(\mathrm{FDR}=7.57 \mathrm{E}-17), \quad$ and $M d k$ $(\mathrm{FDR}=1.75 \mathrm{E}-03)$ were reduced to 77,80 , and $63 \%$ in Ulk4 mutants. Meanwhile, four hyper-anxious genes Gria1 $(123 \%$, FDR $=2.65 \mathrm{E}-14)$, Syngap1 $(114 \%, \mathrm{FDR}=$ 1.06E-08), Npy2r (213\%, FDR $=0.001)$, and Ptpra $(111 \%$, $\mathrm{FDR}=0.002$ ) were significantly increased. Therefore deletion of the Ulk4 gene is associated with imbalanced expression of hyper-anxious and hypo-anxious genes (Table 1).

\section{Validation of the Ulk4 target gene expression}

Whole-genome RNA sequencing is one of the most sensitive methods to detect transcriptome changes at the genome level. Here, we carried out quantitative RT-PCR on six selected Ulk4 targets, the downregulated Pvalb, $M d k$, and Slc38a3, and upregulated Slc38a1, Slc38a2, and Gng2. The transcription of Slc38a1, Slc38a2, and Gng2 was increased to 115,122 , and $127 \%$, respectively, in the Ulk4 vs. WT counterparts, but was statistically not significant (Fig. 5, $p>0.05$ ). However, the Pvalb, Mdk, and Slc38a3 transcripts were reduced to $46.9,58.7$, and $57.8 \%$ (Fig. 5, $n=4$ each, $p<0.05$ ), respectively, in the Ulk4 mutants vs. WT controls. Of particular relevance is the significant reduction of Pvalb transcripts for parvalbumin, which marks a major subgroup of GABA inhibitory interneurons. Parvalbumin deficiency is a landmark neuropathology of schizophrenia ${ }^{22}$ and is associated with mouse model of $\mathrm{ASD}^{23}$. 

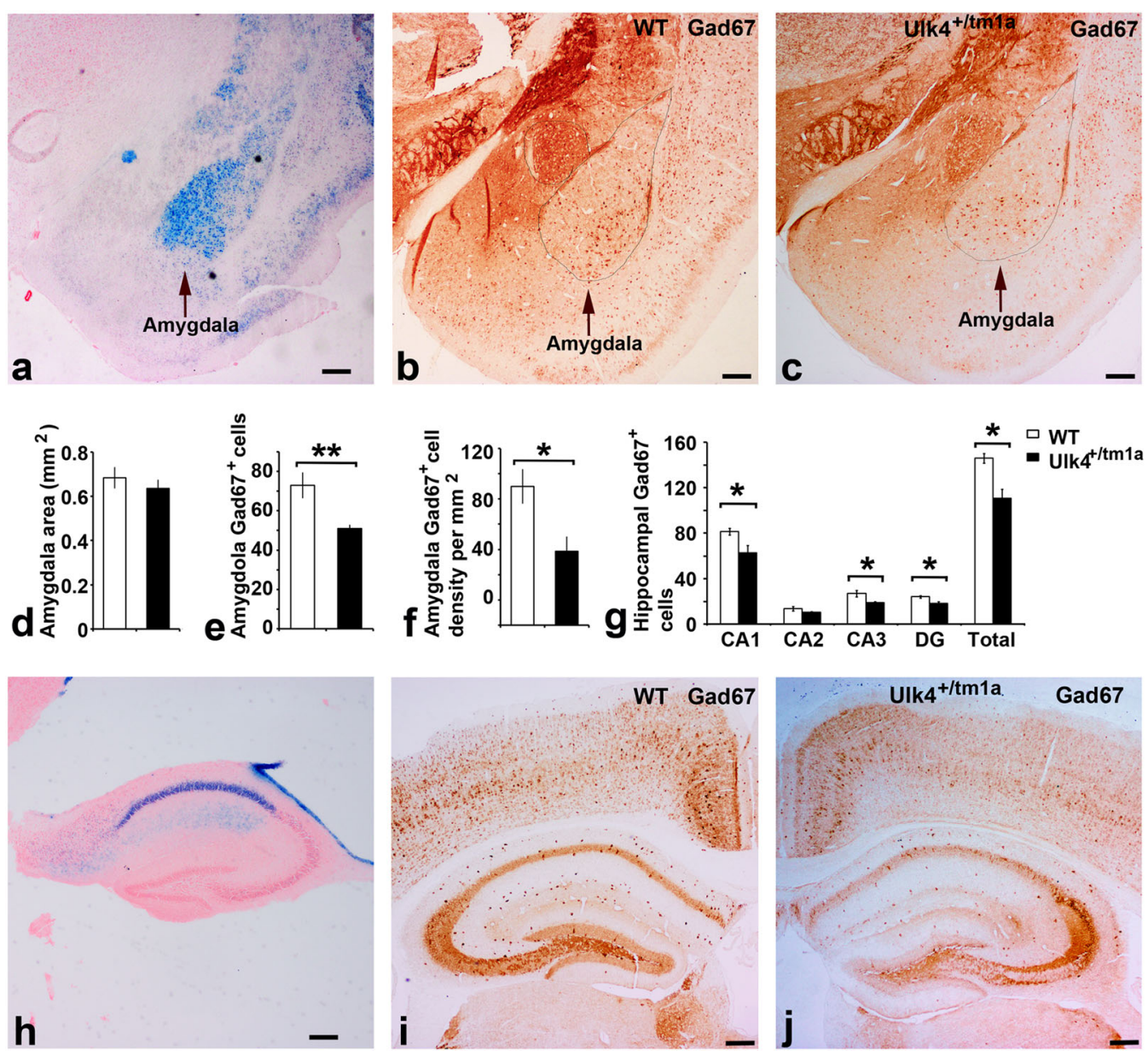

Fig. 4 Reduced Gad $67^{+}$cells in the amygdala and hippocampus of Ulk4 $\mathbf{4}^{+/ t m 1 a}$ mice. X-gal staining showed Ulk4 expression in the basolateral amygdala (a) and hippocampus (b). Anti-Gad67 immunohistochemistry was carried out on brain section of five female WT and five Ulk4 $4^{+/ t m 1 a}$ female mice at 2 months. The Gad67 ${ }^{+}$cells were quantified from the equivalent regions as outlined in WT $\left(0.68 \pm 0.05 \mathrm{~mm}^{2}, n=5\right)$ and Ul/k4 $4^{+/ t m 1 a}(0.64 \pm 0.04$

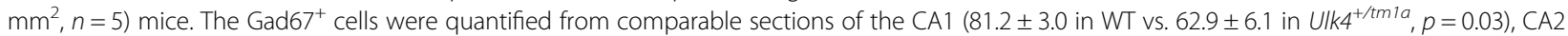
$\left(13.7 \pm 1.9\right.$ in WT vs. $10.3 \pm 0.7$ in Ulk4 $\left.{ }^{+/ t m 1 a}, p=0.13\right)$, CA3 (27.0 \pm 2.8 in WT vs. $19.0 \pm 0.9$ in Ulk4 $\left.{ }^{+/ t m 1 a}, p<0.02\right)$, DG $(24.1 \pm 1.2$ vs. 18.4 $\pm 1.4, p<0.02)$, WT hippocampus $(146.0 \pm 4.3)$ and Ulk4 ${ }^{+/ t m l a}$ hippocampus $(110.6 \pm 8.2, p<0.01)$ and statistically analyzed $(\mathbf{g})$, showing significant reduction of

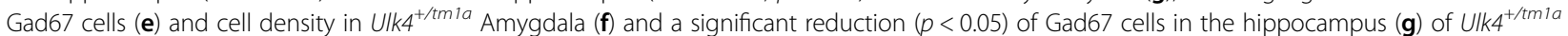
mice. Bar $=200 \mu \mathrm{m}$ in $\mathbf{a}, \mathbf{b}, \mathbf{c}, \mathbf{h}, \mathbf{i}, \mathbf{j} .{ }^{*} p<0.05 ;{ }^{* *} p<0.01$

\section{Discussion}

Previously, we showed that ULK4 was expressed in GABA neurons and ULK4 CNVs were found in neurodevelopmental and neuropsychiatric disorders ${ }^{7,8}$. In this study, we report the first evidence that Ulk4 regulates GABAergic signaling in the brain, and Ulk4 heterozygous mice display anxiety-related phenotype, a prominent component of many neuropsychiatric diseases.

The ULK4 CNVs occur at a rate of $0.14 \%(16 / 11,633)$ from two previous studies ${ }^{7,8}$, lower than NRXN1 CNVs as a major target for schizophrenia $(0.18-0.63 \%)^{24-26}$, and ASD $(0.45 \%)^{27,28}$. The ULK4 mutation rate may rise when the whole-exome or genome are sequenced. However, the clinical features of $U L K 4$ CNVs are pleiotropic, which include schizophrenia, ASD, bipolar disorder ${ }^{7}$, developmental delay, severe language delay, learning difficulties, and behavioral disorder ${ }^{8}$. Therefore, comprehensive behavioral tests were performed on the $U l k 4^{+/ t m 1 a}$ mice in this study. Ulk $4^{+/ t m 1 a}$ mice exhibit no obvious schizophrenia-related or depression-related phenotype, as they did not show statistic difference from WT littermates in ASR, PPI, PST, or TST.

However, $U l k 4^{+/ t m l a}$ mice display anxiety-related behavior in a number of behavioral paradigms. Both $U l k 4^{+/ t m l a}$ male and female mice spent significantly less time on the open arms but more time in the closed arms in the EPM test, a well-recognized measure of anxietyrelated behavior. Ulk $4^{+/ t m l a}$ male mice interacted less with the novel vs. familiar animal, indicative of a deficit in social novelty preference possibly due to social neo- 
Table 1 Ulk4 regulates GABAergic signaling, hyper-anxious, and hypo-anxious genes

\begin{tabular}{|c|c|c|c|c|c|c|}
\hline & WT (FPKM) & Ulk4 mutants (FPKM) & Average mutant/WT & Up-down (mutant/WT) & $p$-value & FDR \\
\hline \multicolumn{7}{|c|}{ GABA subtypes } \\
\hline Pvalb (39\%) & $28.7 \pm 2.9$ & $18.0 \pm 3.0$ & 0.63 & Down & 4.33E-07 & $9.01 \mathrm{E}-06$ \\
\hline Sst (23\%) & $382.4 \pm 22.8$ & $324.9 \pm 11.9$ & 0.85 & Down & 7.97E-10 & $2.61 \mathrm{E}-08$ \\
\hline Cck (5\%) & $437.7 \pm 3.8$ & $358.0 \pm 1.9$ & 0.82 & Down & $2.52 \mathrm{E}-17$ & $1.80 \mathrm{E}-15$ \\
\hline Npy (8\%) & $257.1 \pm 9.9$ & $196.8 \pm 21.4$ & 0.77 & Down & 1.19E-14 & $6.84 \mathrm{E}-13$ \\
\hline Nos3 $(<1 \%)$ & $4.6 \pm 0.4$ & $3.1 \pm 0.6$ & 0.67 & Down & $1.09 \mathrm{E}-04$ & $1.35 \mathrm{E}-03$ \\
\hline Calb2 (24\%) & $11.2 \pm 0.3$ & $16.3 \pm 1.8$ & 1.45 & Up & 5.29E-05 & 7.07E-04 \\
\hline Vip (11\%) & $18.9 \pm 1.3$ & $23.2 \pm 3.4$ & 1.23 & Up & 4.04E-03 & 3.05E-02 \\
\hline \multicolumn{7}{|c|}{ GABAergic synapse pathway } \\
\hline Gng4 & $47 \pm 3.1$ & $40.1 \pm 4.3$ & 0.85 & Down & $5.26 \mathrm{E}-05$ & 7.04E-04 \\
\hline Gng7 & $142.6 \pm 15.9$ & $107.8 \pm 33.4$ & 0.76 & Down & $5.00 \mathrm{E}-53$ & $1.82 \mathrm{E}-50$ \\
\hline S/c38a3 & $42.5 \pm 5.6$ & $31.5 \pm 7.4$ & 0.74 & Down & $2.37 \mathrm{E}-10$ & 8.49E-09 \\
\hline Slc38a5 & $7.1 \pm 1.4$ & $3.7 \pm 1.4$ & 0.53 & Down & $8.81 \mathrm{E}-06$ & $1.43 \mathrm{E}-04$ \\
\hline Adcy9 & $9.7 \pm 0.8$ & $13.4 \pm 2.2$ & 1.39 & Up & $1.91 \mathrm{E}-13$ & $9.77 \mathrm{E}-12$ \\
\hline Cacnalc & $7.2 \pm 0.4$ & $8.6 \pm 0.5$ & 1.19 & Up & $3.57 \mathrm{E}-05$ & $4.98 \mathrm{E}-04$ \\
\hline Gabral & $78.4 \pm 8.5$ & $95 \pm 8.7$ & 1.21 & Up & $2.55 \mathrm{E}-22$ & $2.60 \mathrm{E}-20$ \\
\hline Gabra3 & $36.5 \pm 2.5$ & $44.4 \pm 3$ & 1.21 & Up & $1.24 \mathrm{E}-08$ & 3.39E-07 \\
\hline Gabra4 & $32.7 \pm 1.6$ & $38.8 \pm 1.5$ & 1.19 & Up & $3.91 \mathrm{E}-07$ & $8.21 \mathrm{E}-06$ \\
\hline Gabra5 & $35.5 \pm 0.3$ & $43.3 \pm 4.9$ & 1.22 & Up & 2.03E-06 & $3.76 \mathrm{E}-05$ \\
\hline Gabrb3 & $74.8 \pm 5.8$ & $95.5 \pm 8.9$ & 1.28 & Up & $6.62 E-35$ & $1.29 \mathrm{E}-32$ \\
\hline Gng2 & $85.6 \pm 2$ & $103.2 \pm 10.7$ & 1.20 & Up & $1.35 \mathrm{E}-16$ & $8.93 \mathrm{E}-15$ \\
\hline Hap1 & $15.7 \pm 2.2$ & $26.6 \pm 5$ & 1.70 & Up & 4.26E-24 & $4.92 \mathrm{E}-22$ \\
\hline Nsf & $174.4 \pm 15.1$ & $214.9 \pm 9.8$ & 1.23 & Up & $3.11 \mathrm{E}-38$ & 7.03E-36 \\
\hline Slc38a1 & $21.7 \pm 0.8$ & $28.1 \pm 1$ & 1.30 & Up & $5.89 \mathrm{E}-18$ & $4.41 \mathrm{E}-16$ \\
\hline Slc38a2 & $21.3 \pm 0.6$ & $33.6 \pm 8.4$ & 1.58 & Up & $5.01 \mathrm{E}-33$ & $9.04 \mathrm{E}-31$ \\
\hline \multicolumn{7}{|c|}{ Hypo-anxious genes } \\
\hline Atpla2 & $228.0 \pm 18.7$ & $175.47 \pm 19.7$ & 0.77 & Down & $1.28 \mathrm{E}-117$ & $1.40 \mathrm{E}-114$ \\
\hline Ptn & $178.6 \pm 26.3$ & $142.9 \pm 33.5$ & 0.80 & Down & $9.28 \mathrm{E}-19$ & $7.57 \mathrm{E}-17$ \\
\hline Mdk & $22.8 \pm 3.9$ & $14.5 \pm 6.1$ & 0.63 & Down & 1.47E-04 & $1.75 E-03$ \\
\hline Plcb4 & $11.4 \pm 2.4$ & $14.9 \pm 2.5$ & 1.31 & Up & $1.50 \mathrm{E}-05$ & $2.28 \mathrm{E}-04$ \\
\hline Apoe & $1085.4 \pm 162.0$ & $1127.2 \pm 46.3$ & 1.04 & Up & $1.56 \mathrm{E}-05$ & $2.36 \mathrm{E}-04$ \\
\hline Cacnale & $15.1 \pm 3.5$ & $17.0 \pm 0.3$ & 1.13 & Up & $2.75 \mathrm{E}-04$ & $3.06 \mathrm{E}-03$ \\
\hline Adra2a & $8.4 \pm 1.1$ & $10.7 \pm 1.7$ & 1.28 & Up & $3.72 \mathrm{E}-04$ & $3.97 \mathrm{E}-03$ \\
\hline \multicolumn{7}{|c|}{ Hyper-anxious genes } \\
\hline Ncaml & $96.0 \pm 4.7$ & $82.8 \pm 8.5$ & 0.86 & Down & $4.78 \mathrm{E}-12$ & $2.09 \mathrm{E}-10$ \\
\hline Gria 1 & $48.4 \pm 6.7$ & $59.4 \pm 8.3$ & 1.23 & Up & 4.14E-16 & $2.65 E-14$ \\
\hline Syngap 1 & $106.8 \pm 10.0$ & $121.9 \pm 27.1$ & 1.14 & Up & 3.03E-10 & $1.06 \mathrm{E}-08$ \\
\hline Npy2r & $0.9 \pm 0.3$ & $1.9 \pm 0.6$ & 2.13 & Up & 1.15E-04 & $1.41 \mathrm{E}-03$ \\
\hline Ptpra & $56.6 \pm 2.2$ & $62.9 \pm 2.4$ & 1.11 & Up & $1.98 \mathrm{E}-04$ & $2.29 \mathrm{E}-03$ \\
\hline
\end{tabular}



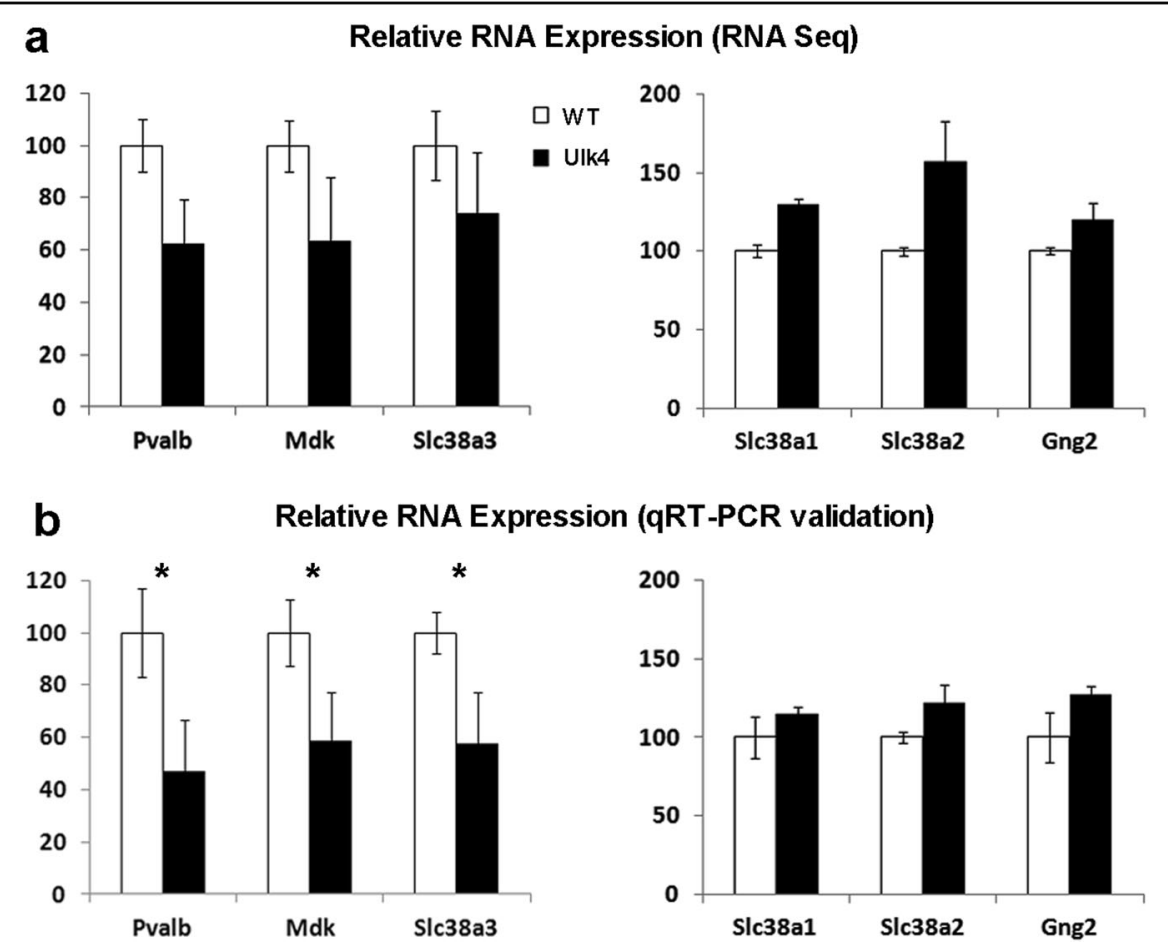

Fig. 5 Altered gene expression identified by whole-genome RNA sequencing (a), and validated by quantitative RT-PCR (b). RNA sequencing was carried out in three pairs of P12 WT and Ulk4 ${ }^{\text {tmla/tmla }}$ cortex, and quantitative RT-PCR in four pairs of cortical RNA (b). Whereas S/c38a1, S/c38a2, and Gng2 genes showed increased mRNA transcripts by both methods, the Pvalb, Mdk, and S/c38a3 gene transcription were significantly reduced $\left({ }^{*} p\right.$ $<0.05)$

phobia. Ulk $4^{+/ t m 1 a}$ females failed to acclimatize the novel open field arena at the end phase of the test, seen as a lack of increase in grooming behavior, and buried significantly more marbles in the MBT. The lack of acclimatization is likely due to the enhanced anxiety of these animals in a novel environment and increased marble burying is a well-recognized behavior associated with neo-phobia/anxiety-related behavior. Thus, both male and female $U l k 4$ mutants exhibit anxiety-related behavior in several behavioral paradigms, although some sex-related differences were noted. The reason for sex differences in some but not all of the behavioral tests is unknown; however, Ulk4 may regulate neuro-hormone release and/or activity, as Ulk4 is expressed also in brain regions mediating homeostatic control, such as the hypothalamus and neurosecretory circumventricular nuclei $^{29}$, which may result in sex-related differences in behavior under certain conditions. Despite the subtle differences in responses between males and females in individual tests, taken together, the behavioral data demonstrate an anxiety-like phenotype in $U l k 4^{+/ t m 1 a}$ mice.

Consistent with the behavioral changes, three previously identified hypo-anxious genes (Atp1a2, Ptn, and Mdk) were downregulated, and four hyper-anxious genes
(Gria1, Syngap1, Npy2r, and Ptpra) were significantly upregulated, suggesting that $U l k 4$ lesion disturbs balanced expression of hyper-anxious and hypo-anxious genes in the brain $^{2}$. Ulk4 also plays a role in neurogenesis ${ }^{8-10}$, which is in agreement with the hypothesis that anxious phenotype is associated with neurodevelopmental changes $^{2}$.

In addition, the G-protein-coupled signaling appears also disrupted, as Gng4, Gng7, Adcy9, and Kcnj6 were dysregulated in Ulk4 mutants. Gng4 and Gng7 are mediators of GPCR signaling linked to fear and anxiety, and Gng4 expression was inversely correlated with fear and anxiety in the mouse model of post-traumatic stress disorder $^{30}$. Gng7 was significantly downregulated in the amygdala of Itpka $\mathrm{KO}$ mice which exhibited fear-related and anxiety-related behaviors ${ }^{31}$. Subtle changes in Gng7 expression also impacted anxiety and aggressive behaviors in transgenic animals ${ }^{32}$ and in chronic social-defeat stress leading to depressive and anxious states ${ }^{33}$. ADCY9, involved in cAMP production, was previously identified as a risk factor for mood disorders ${ }^{34,35}$. However, the relevance of Kcnj6 (encoding a G-protein activated inward rectifier potassium channel) dysregulation is less certain. Kcnj6 is located on human Chr21, and trisomy Kcnj6 transgenic mice display some of the Down syndrome-like 
neurological abnormalities, but not anxiety-related indices $^{36}$.

Overwhelming evidence suggests that abnormal GABA transmission contributes to the pathophysiology of anxiety disorders in humans $\mathrm{s}^{37,38}$. For instance, studies using nuclear imaging techniques revealed diminished central GABA and GABA receptor levels in patients with panic disorder, generalized anxiety disorder, and posttraumatic stress disorder ${ }^{39}$. The GAD2 polymorphisms were shown as a risk factor for anxiety disorders ${ }^{40}$. Genetic variations $^{41}$ and reduced GAD67 expression in bipolar and schizophrenia were also well documented ${ }^{42,43}$. Similarly, $\mathrm{Gad1}^{+/-}$male mice also exhibit reduced $\mathrm{Gad}^{+} 7^{+}$neurons, with disturbed characteristics of depression and anxiety $^{44}$. Considering significant decreases in Gad67 neurons in the hippocampus and basolateral amygdala of $U l k 4^{+/ t m l a}$ mice, and the correlating functions of GAD1 in humans, it will be interesting to investigate if polymorphisms of the ULK4 link to anxiety disorders in patients.

GABA transmission in the amygdala is considered particularly important in controlling fear and anxiety. We confirmed high Ulk4 expression in the basolateral amygdala and hippocampus using X-gal staining. Anti-Gad67 staining revealed significant reduction of $\mathrm{Gad} 67^{+}$neurons in hippocampus and amygdala of the $U l k 4^{+/ t m 1 a}$ mice. Interestingly, high $\beta$-gal activity is located in the medial aspect of the basolateral amygdala, whereas $\mathrm{Gad} 67^{+}$cells are more abundant in the lateral aspect of the basolateral amygdala. In the hippocampus, strong X-gal staining is present in the CA1, while $\mathrm{Gad} 67^{+}$cells are reduced in all hippocampal regions. These anatomic differences in the patterns of X-gal and Gad67 staining may be due to alternative splicing of the Ulk4 gene, for example, Ulk4 isoforms may be started after exon 6 , which will not be revealed by X-gal staining. Alternatively, Ulk4 protein may also be expressed in other neuronal types, other than $\mathrm{Gad}^{+} 7^{+}$neurons, such as $\mathrm{Gad}^{+} 5^{+}$neurons or other neuronal subtypes. We cannot rule out that the change in Gad67 ${ }^{+}$cell number in Ulk4 mutants may also be an indirect or compensatory consequence of Ulk4 deletion during development and not be related to loss of Ulk4 function in GABAergic neurons. Regardless of the precise mechanism, the data herein demonstrate that $U l k 4^{+/ t m 1 a}$ mice exhibit reduced cell number of $\mathrm{Gad}_{67}{ }^{+}$neurons and thus possibly altered GABAergic neurotransmission in the BLA and hippocampus that may underlie the behavioral changes observed.

In humans benzodiazepines are the most widely prescribed anxiolytic drugs acting through GABA receptors $^{38}$. In rodents, administration of $\mathrm{GABA}_{\mathrm{A}} \mathrm{R}$ agonists, including muscimol, also decreases the anxiety level ${ }^{45,46}$. GABA is synthesized from glutamate, which is transported into cells by membrane proteins. It is worth to note that four sodium-dependent amino acid transporters were dysregulated in the mutants. Whereas expression of the Slc38a3 and Slc38a5 were reduced, Slc38a1 and Slc38a2 expression were increased. These alterations were consistent with reported anxiety phenotype. For example, Slc38a1 was overexpressed in MeCP2 mutants, a mouse models for RETT syndrome with communication deficit, motor impairments, hand stereotypies ${ }^{47}$, and anxiety ${ }^{48}$. Slc38a3 is an anxiety-related response locus QTL15 in rat. Slc38a3 and Gng4 expression were altered in EFhd2 knockout mouse associated with anxiety and alcohol addiction $^{49}$. Slc38a5 was one of the two genes dysregulated in $\mathrm{Rail}^{+/-}$mice with epilepsy, mental retardation, and anxiety disorder ${ }^{50}$.

There are $>12$ subtypes of GABA interneurons in mammalian brain ${ }^{21}$. Transcriptome analyses in this report showed significant reduction of many GABA interneuron subtypes in the Ulk4 hypomorphs, including Pvalb, Sst, Cck, Npy, and Nos3, which constituted three quarters of total GABA interneurons in normal mice. Parvalbumin, the most abundant subtype whose deficiency is a fundamental pathology in neurodevelopmental disorders ${ }^{22,23}$, is significantly downregulated in $U l k 4$ mutants.

Alterations in the density and number of GABAergic neurons in the brain occur throughout postnatal development $^{51-53}$. It is not possible to identify exactly when Ulk4 deletion would specifically alter GABAergic cell density and/or function but rather it is likely that alterations occur along the neurodevelopmental pathway. Despite this, RNA sequencing was done on P12 Ulk $4^{\text {tmlal }}$ ${ }^{t m 1 a}$ and WT mice which reveal alterations in expression of GABA neuronal subtypes, and receptor subtypes. Furthermore, in 2-month old, we show again regional reduction of $\mathrm{Gad} 7^{+}$neurons in 2-month mice, the time correlating with behavioral testing.

GABA interneurons play their inhibition roles largely via brain $\mathrm{GABA}_{\mathrm{A}}$ Rs and $\mathrm{GABA}_{\mathrm{B}}$ Rs. The fast short-lasting "phasic" inhibition is typically generated by the activation of postsynaptic $\mathrm{GABA}_{\mathrm{A}}$ Rs following action potentials in presynaptic interneurons, and the "tonic" extra-synaptic inhibition is activated by ambient GABA in the extracellular space through molecularly and functionally specialized $\mathrm{GABA}_{\mathrm{A}}$ Rs. Remarkably the postsynaptic GABAergic signaling was also disrupted in Ulk4 mutants, and this included significant upregulation of $G_{A B A} R$ subunits Gabra1, Gabra3, Gabra4, Gabra5, and Gabrb3. Changes in these receptor subunits are consistent with the anxiety phenotype. For example, the human GABRA5 and GABRB3 are located in the $15 \mathrm{q} 11.2-\mathrm{q} 13$ region, and maternal duplications of 15q11.2-q13 leads to neurodevelopmental disorders including ASD, and their clinical symptoms often include anxiety, emotional lability, tantrums, and hyperactivity ${ }^{54}$. Although deletion of Gabra1 in amygdala did not affect anxiety behavior ${ }^{55}$, Gabra3- 
mediated tonic inhibition in the amygdala was essential in regulating fear and anxiety. The Gabra3-selective benzodiazepine site agonist and anxiolytic compound TP003 increased tonic currents and markedly dampen excitability in BLA principal cells ${ }^{56}$. Gabra4 was involved in fear extinction learning, and knockout of the extrasynaptic $\mathrm{GABA}_{\mathrm{A}}$ Rs facilitated fear extinction ${ }^{57}$. Altered expression also included Nsf, which was shown to downregulate $\mathrm{GABA}_{\mathrm{A}} \mathrm{Rs}$ with $\mathrm{PKC}^{58}$. We, however, cannot rule out that $U l k 4$ deletion may affect GABAergic neurotransmission in other brain regions, and Ulk4 may be also expressed in other neurons such as glutamatergic neurons. Examination of effects in other neurons will be the focus of follow-up studies.

Sex bias is common in brain disorders and sex is known to influence GABAergic transmission and anxiety-related behavior $^{59}$. The data herein demonstrate that Llk4 deletion alters various GABAergic parameters; however, assessment of such parameters was not conducted in a sufficient number of males and females separately to enable sex differences to be analyzed at this level. Despite subtle sex-related differences are observed in different behavioral paradigms, the strongest phenotype is the increased anxiety from plus maze test, which are consistent in both male and female mutants. Thus, the data suggest that Ulk4 deletion modulates brain development and results in GABAergic changes which may underlie the anxiety-related phenotype.

In summary, we have shown for the first time that $U l k 4$ haploinsufficiency in mice leads to increased anxietyrelated behavior with disturbed GABAergic signaling. Ulk4 is involved in the maintenance of the excitation/ inhibition balance, which is commonly disturbed in neurodevelopmental and neuropsychiatric disorders. Therefore, regulation of the ULK4 activity may present an alternative route of drug exploration for neurodevelopmental and neuropsychiatric illness.

\section{Acknowledgements}

Authors wish to acknowledge the Science Foundation Ireland (grant number 09/SRC/B1794s1 and 13/IA/1787 to S.S.), National University of Ireland Galway (grant number RSU002 to S.S.), and Molecular Medicine Ireland Clinical \& Translational Research Scholars Programme for funding the research; and the staff of the Bio-Resources Unit, in particular Drs. Yolanda Garcia and Cathal O'Flatharta, for the support and assistance of experimental procedure. The authors would also like to thank the KOMP Repository (www.komp.org), the Mouse Biology Program (www.mousebiology.org) at the University of California Davis for Ulk4t/tmla mice.

\footnotetext{
Author details

${ }^{1}$ Regenerative Medicine Institute, School of Medicine, National University of Ireland Galway, Galway, Ireland. ${ }^{2}$ Physiology, School of Medicine, Galway Neuroscience Centre and Centre for Pain Research, National University of Ireland Galway, Galway, Ireland. 'Department of Physiology, College of Life Science, Hebei Normal University, Shijiazhuang, China. ${ }^{4}$ National Center for Protein Sciences, Beijing Proteome Research Center, National Engineering Research Center for Protein Drugs, Beijing Institute of Radiation Medicine, Beijing, China. ${ }^{5}$ School of Biomedical Sciences, University of Leeds, Leeds, UK
}

Conflict of interest

The authors declare that they have no conflict of interest.

\section{Publisher's note}

Springer Nature remains neutral with regard to jurisdictional claims in published maps and institutional affiliations.

Supplementary Information accompanies this paper at https://doi.org/ 10.1038/s41398-017-0091-5.

Received: 23 June 2017 Revised: 9 October 2017 Accepted: 30 November 2017

Published online: 02 February 2018

\section{References}

1. Wittchen, H. U. et al. The size and burden of mental disorders and other disorders of the brain in Europe 2010. Eur. Neuropsychopharm. 21, 655-679 (2011).

2. Viggiano, A., Cacciola, G., Widmer, D. A. J. \& Viggiano, D. Anxiety as a neurodevelopmental disorder in a neuronal subpopulation: evidence from gene expression data. Psychiatry Res. 228, 729-740 (2015).

3. Soghomonian, J. J. \& Martin, D. L. Two isoforms of glutamate decarboxylase: why? Trends Pharmacol. Sci. 19, 500-505 (1998).

4. Fenalti, G. et al. GABA production by glutamic acid decarboxylase is regulated by a dynamic catalytic loop. Nat. Struct. Mol. Biol. 14, 280-286 (2007).

5. Kash, S. F. et al. Epilepsy in mice deficient in the $65-\mathrm{kDa}$ isoform of glutamic acid decarboxylase. Proc. Natl. Acad. Sci. USA 94, 14060-14065 (1997).

6. Stork, O. et al. Postnatal development of a GABA deficit and disturbance of neural functions in mice lacking GAD65. Brain Res. 865, 45-58 (2000).

7. Lang, B. et al. Recurrent deletions of ULK4 in schizophrenia: a gene crucial for neuritogenesis and neuronal motility. J. Cell. Sci. 127(Pt 3), 630-640 (2014).

8. Liu, M. et al. Ulk4 regulates neural stem cell pool. Stem Cells 34, 2318-2331 (2016).

9. Lang, B. et al. Control of cortex development by ULK4, a rare risk gene for mental disorders including schizophrenia. Sci. Rep. 6, 31126 (2016).

10. Liu, M., Xu, P., O'Brien, T. \& Shen, S.. Multiple roles of Ulk4 in neurogenesis and brain function. Neurogenesis 4, (2017).

11. Liu, M. et al. Ulk4 is essential for ciliogenesis and CSF flow. J. Neurosci. 36, 7589-7600 (2016).

12. Skarnes, W. C. et al. A conditional knockout resource for the genome-wide study of mouse gene function. Nature 474, 337-U361 (2011).

13. Crawley, J. N. Designing mouse behavioral tasks relevant to autistic-like behaviors. Ment. Retard. Dev. Disabil. Res. Rev. 10, 248-258 (2004).

14. Nadler, J. J. et al. Automated apparatus for quantitation of social approach behaviors in mice. Genes. Brain Behav. 3, 303-314 (2004).

15. Kerr, D. M., Gilmartin, A. \& Roche, M. Pharmacological inhibition of fatty acid amide hydrolase attenuates social behavioural deficits in male rats prenatally exposed to valproic acid. Pharmacol. Res. 113, 228-235 (2016).

16. Kerr, D. M., Downey, L., Conboy, M., Finn, D. P. \& Roche, M. Alterations in the endocannabinoid system in the rat valproic acid model of autism. Behav. Brain Res. 249, 124-132 (2013).

17. Deacon, R. M. J. Digging and marble burying in mice: simple methods for in vivo identification of biological impacts. Nat. Protoc. 1, 122-124 (2006).

18. Njunge, K. \& Handley, S. L. Evaluation of marble-burying behavior as a model of anxiety. Pharmacol. Biochem. Behav. 38, 63-67 (1991).

19. Walf, A. A. \& Frye, C. A. The use of the elevated plus maze as an assay of anxiety-related behavior in rodents. Nat. Protoc. 2, 322-328 (2007).

20. MacKenzie-Graham, A. et al. A multimodal, multidimensional atlas of the C57BL/6J mouse brain. J. Anat. 204, 93-102 (2004).

21. Gonchar, Y., Wang, Q. \& Burkhalter, A. Multiple distinct subtypes of GABAergic neurons in mouse visual cortex identified by triple immunostaining. Front. Neuroanat. 1, 3 (2007).

22. Lewis, D. A., Hashimoto, T. \& Volk, D. W. Cortical inhibitory neurons and schizophrenia. Nat. Rev. Neurosci. 6, 312-324 (2005)

23. Woehr, M.et al Lack of parvalbumin in mice leads to behavioral deficits relevant to all human autism core symptoms and related neural morphofunctional abnormalities. Transl. Psychiatry 5, (2015). 
24. Kirov, G. et al. Comparative genome hybridization suggests a role for NRXN1 and APBA2 in schizophrenia. Human. Mol. Genet. 17, 458-465 (2008).

25. Rujescu, D. et al. Disruption of the neurexin 1 gene is associated with schizophrenia. Human. Mol. Genet. 18, 988-996 (2009).

26. Marshall, C. R. et al. Contribution of copy number variants to schizophrenia from a genome-wide study of 41,321 subjects. Nat. Genet. 49, 27-35 (2017).

27. Glessner, J. T. et al. Autism genome-wide copy number variation reveals ubiquitin and neuronal genes. Nature 459, 569-573 (2009).

28. Pinto, D. et al. Convergence of genes and cellular pathways dysregulated in autism spectrum disorders. Am. J. Human. Genet. 94, 677-694 (2014).

29. Dominguez, L., Schlosser, G. \& Shen, S. Expression of a novel serine/threonine kinase gene, Ulk4, in neural progenitors during Xenopus laevis forebrain development. Neuroscience 290, 61-79 (2015)

30. Muhie, S. et al. Brain transcriptome profiles in mouse model simulating features of post-traumatic stress disorder. Mol. Brain 8, 14 (2015).

31. Chung, S. et al. The role of inositol 1,4,5-trisphosphate 3-kinase A in regulating emotional behavior and amygdala function. Sci. Rep. 6, 23757 (2016).

32. Schwindinger, W. F. et al. Loss of G protein gamma(7) alters behavior and reduces striatal alpha(olf) level and CAMP production. J. Biol. Chem. 278, 6575-6579 (2003).

33. Azzinnari, D. et al. Mouse social stress induces increased fear conditioning, helplessness and fatigue to physical challenge together with markers of altered immune and dopamine function. Neuropharmacology 85, 328-341 (2014).

34. Toyota, T. et al. Molecular analysis, mutation screening, and association study of adenylate cyclase type 9 gene (ADCY9) in mood disorders. Am. J. Med. Genet. 114, 84-92 (2002).

35. Muglia, P. et al. Genome-wide association study of recurrent major depressive disorder in two European case-control cohorts. Mol. Psychiatry 15, 589-601 (2010).

36. Cooper, A. et al. Trisomy of the G protein-coupled $\mathrm{K}+$ channel gene, $\mathrm{K} c n j 6$, affects reward mechanisms, cognitive functions, and synaptic plasticity in mice. Proc. Natl. Acad. Sci. USA 109, 2642-2647 (2012).

37. Lydiard, R. B. The role of GABA in anxiety disorders. J. Clin. Psychiatry 64, 21-27 (2003).

38. Nemeroff, C. B. The role of GABA in the pathophysiology and treatment of anxiety disorders. Psychopharmacol. Bull. 37, 133-146 (2003).

39. Millan, M. J. The neurobiology and control of anxious states. Prog. Neurobiol. 70, 83-244 (2003)

40. Unschuld, P. G. et al. Polymorphisms in the GAD2 gene-region are associated with susceptibility for unipolar depression and with a risk factor for anxiety disorders. Am. J. Med. Genet. B Neuropsychiatr. Genet. 150B, 1100-1109 (2009).

41. Addington, A. M. et al. GAD1 (2q31.1), which encodes glutamic acid decarboxylase (GAD67), is associated with childhood-onset schizophrenia and cortical gray matter volume loss. Mol. Psychiatry 10, 581-588 (2005).

42. Heckers, S. et al. Differential hippocampal expression of glutamic acid decarboxylase 65 and 67 messenger RNA in bipolar disorder and schizophrenia Arch. Gen. Psychiatry 59, 521-529 (2002)
43. Hashimoto, T. et al. Gene expression deficits in a subclass of GABA neurons in the prefrontal cortex of subjects with schizophrenia. J. Neurosci. 23, 6315-6326 (2003).

44. Sandhu, K. V. et al. Glutamic acid decarboxylase 67 haplodeficiency impairs social behavior in mice. Genes. Brain Behav. 13, 439-450 (2014).

45. Zarrindast, M. R., Rostami, P. \& Sadeghi-Hariri, M. GABA(A) but not GABA(B) receptor stimulation induces antianxiety profile in rats. Pharmacol. Biochem. Behav. 69, 9-15 (2001)

46. Lippa, A. et al. Selective anxiolysis produced by ocinaplon, a GABA(A) receptor modulator. Proc. Natl. Acad. Sci. USA 102, 7380-7385 (2005)

47. Jin, L. W. et al. Dysregulation of glutamine transporter SNAT1 in Rett syndrome microglia: a mechanism for mitochondrial dysfunction and neurotoxicity. J. Neurosci. 35, 2516-2529 (2015).

48. Barnes, K. V. et al. Anxiety-like behavior in Rett syndrome: characteristics and assessment by anxiety scales. J. Neurodev. Disord. 7, 30 (2015).

49. Mielenz D. et al. EFhd2/Swiprosin-1 is a common genetic determinator for sensation-seeking/low anxiety and alcohol addiction. Mol. Psychiatry https:// doi.org/10.1038/mp.2017.63 (2017).

50. Burns, B. et al. Rai1 haploinsufficiency causes reduced Bdnf expression resulting in hyperphagia, obesity and altered fat distribution in mice and humans with no evidence of metabolic syndrome. Human. Mol. Genet. 19, 4026-4042 (2010).

51. Micheva, K. D. \& Beaulieu, C. Postnatal-development of gaba neurons in the Rat somatosensory barrel cortex-a quantitative study. Eur. J. Neurosci. 7, 419-430 (1995)

52. Bosch, D. \& Ehrlich, I. Postnatal maturation of GABAergic modulation of sensory inputs onto lateral amygdala principal neurons. J. Physiol. 593, 4387-4409 (2015).

53. Qu, G. J., Ma, J., Yu, Y. C. \& Fu, Y. Postnatal development of GABAergic interneurons in the neocortical subplate of mice. Neuroscience 322, 78-93 (2016).

54. Hogart, A., Wu, D., LaSalle, J. M. \& Schanen, N. C. The comorbidity of autism with the genomic disorders of chromosome 15q11.2-q13. Neurobiol. Dis. 38, 181-191 (2010)

55. Heldt, S. A. \& Ressler, K. J. Amygdala-specific reduction of alpha1-GABAA receptors disrupts the anticonvulsant, locomotor, and sedative, but not anxiolytic, effects of benzodiazepines in mice. J. Neurosci. 30, 7139-7151 (2010).

56. Marowsky, A., Rudolph, U., Fritschy, J. M. \& Arand, M. Tonic inhibition in principal cells of the amygdala: a central role for alpha3 subunit-containing GABAA receptors. J. Neurosci. 32, 8611-8619 (2012).

57. Paydar, A. et al. Extrasynaptic GABAA receptors in mediodorsal thalamic nucleus modulate fear extinction learning. Mol. Brain 7, 39 (2014).

58. Chou, W. H. et al. GABA(A) receptor trafficking is regulated by protein kinase $C$ epsilon and the N-ethylmaleimide-sensitive factor. J. Neurosci. $\mathbf{3 0}$, 13955-13965 (2010).

59. Seney, M. The role of genetic sex in affect regulation and expression of GABArelated genes across species. Biol. Psychiatry 73, 108s-109s (2013). 